\title{
High ATP2A2 expression correlates with better prognosis of diffuse astrocytic tumor patients
}

\author{
WEI-QING LI ${ }^{1 *}$, NAN-ZHE ZHONG ${ }^{2 *}$, JIN HE $^{1 *}$, YI-MING LI ${ }^{3}$, LI-JUN HOU $^{3}$, HUI-MIN LIU $^{1}$ \\ CHUN-YAN XIA ${ }^{1}$, LIANG-ZHE WANG ${ }^{1}$ and YI-CHENG LU ${ }^{3}$ \\ Departments of ${ }^{1}$ Pathology, ${ }^{2}$ Orthopedic Oncology and ${ }^{3}$ Neurosurgery, Changzheng Hospital, \\ Second Military Medical University, Shanghai 200003, P.R. China
}

Received September 28, 2016; Accepted March 3, 2017

DOI: 10.3892/or.2017.5528

\begin{abstract}
Novel molecular markers are required for defining subsets of diffuse astrocytic tumor patients with differing prognoses. Here, we examined ATP2A2 expression in 109 human diffuse astrocytic tumor samples (39 grade II diffuse astrocytoma (DA), 19 grade III anaplastic astrocytoma (AA), 51 grade IV glioblastoma) and its correlation with patient clinicopathologic characteristics. ATP2A2 expression significantly correlated with tumor grade and survival $(\mathrm{P}<0.05)$. High ATP2A2 expression was detected in $35.3 \%(18 / 51)$ of glioblastoma patients, compared to $61.5 \%$ (24/39) in grade II, and $52.6 \%(10 / 19)$ in grade III astrocytoma patients $(\mathrm{P}=0.043)$. The median survival was $45 \pm 5.3$ (95\% CI, 34.7-55.3) months in patients with high ATP2A2 expression and 16 \pm 5.0 (95\% CI, 6.3-25.7) months in patients with low ATP2A 2 expression $(\mathrm{P}<0.0001)$. Additionally, high grade astrocytoma patients with high ATP2A2 expression showed longer survival

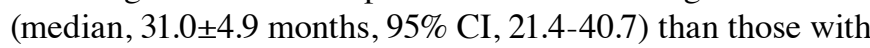

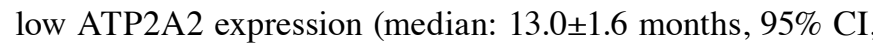
9.9-16.1; $\mathrm{P}=0.027$ ). Furthermore, both ATP2A 2 overexpression and IDH1 mutation were detected in secondary glioblastoma, AA developed from DA and oligodendrogiomas with IDH1 mutation. The MTT assays showed that lentiviral ATP2A2 overexpression significantly suppressed the clonogenic growth of glioblastoma U251MG cells $(\mathrm{P}<0.05)$. Xenografts stably overexpressing ATP2A2 were markedly smaller in size 4 weeks post inoculation $(\mathrm{P}<0.05)$. Our findings identified high ATP2A2 expression in a subset of astrocytoma patients that

Correspondence to: Dr Yi-Ming Li, or Dr Li-Jun Hou, Department of Neurosurgery, Changzheng Hospital, Second Military Medical University, 415 Fengyang Road, Shanghai 200003, P.R. China

E-mail: yiyimig@163.com

E-mail: houlijuncz021@163.com

${ }^{*}$ Contributed equally
\end{abstract}

Key words: astrocytoma, glioblastoma, ATP2A2, oncogenesis, prognosis was associated with better prognosis and ATP2A2 suppressed astrocytoma growth.

\section{Introduction}

Astrocytoma, which originates from star-shaped glial cells, is the most commonly occurring primary brain tumor in adults. According to the World Health Organization (WHO) scheme, it represents a heterogeneous group of diseases with different degrees of malignancy from relatively indolent diffuse astrocytomas to highly aggressive glioblastoma multiforme (GBM) (1). Among these tumors, grade II astrocytomas are characterized by low level proliferative activity, but often recur, and some of the types tend to progress to higher grades of malignancy $(1,2)$. The prognosis of patients with GBM, the most malignant astrocytoma, remains rather dismal. Even with the best standard of care currently available, these patients only have a median life expectancy of $~ 10.6$ months after diagnosis (3). The current standard therapy for GBM patients includes maximal debulking surgery, radiation and adjuvant chemotherapy. Temozolomide, a current chemotherapeutic agent of choice for GBM patients, has yielded only very modest improvements in disease outcomes (4).

Considerable research efforts have been focused on the identification of genetic alterations in GBMs so as to help define subgroups of GBM patients with different prognosis. Several genes, including TP53, PTEN, EGFR and IDH1, are altered in gliomas. The TP53/IDH1 mutation seems to occur early during the development of an astrocytoma (5), whereas the loss or mutation of PTEN and amplification of EGFR are characteristic of primary glioblastomas $(6,7)$. The discovery of these genetic alterations helps the diagnosis of GBM and allows us to better define the prognosis of GBM patients and provides further characterization and understanding of gliomagenesis.

The ATP2A2 gene encodes a protein called sarco (endo) plasmic reticulum $\mathrm{Ca}^{2+}$-ATPase isoform 2 (SERCA2), which transports $\mathrm{Ca}^{2+}$ from the cytosol to the endoplasmic reticulum (ER) lumen. Mutations in ATP2A2 cause Darier's disease, an autosomal dominant skin disorder characterized by loss of adhesion between epidermal cells and abnormal keratinization $(8,9)$. Disturbances of $\mathrm{Ca}^{2+}$ homeostasis have been implicated in the development of many types of tumor, such 
as colon cancer, liposarcoma, leukemia and head and neck squamous cancer (10-15). ATP2A2 heterozygous mutant $\left(A T P 2 A 2^{+/-}\right)$mice showed increased incidence of hyperkeratinized tumors in regions of stratified squamous epithelia, including the oral mucosa, tongue, esophagus, palate, skin, genitalia, and non-glandular mucosa of the stomach (16). The role of ATP2A2 in the oncogenesis of astrocytomas has not been elucidated. We hypothesized that, given the importance of $\mathrm{Ca}^{2+}$ homeostasis in maintaining the physiological processes, the expression of ATP2A 2 could be altered in astrocytomas and the definition of such alterations may, as a prognostic marker, help identify subgroups of patients with a distinct prognosis, or, as a diagnostic marker, help distinguish astrocytomas of different grades.

Although ATP2A2 has recently been implicated in certain cancer types $(19,20)$, no information is available on its expression in human astrocytoma tissues. In this study, we examined the expression of ATP2A2 in 109 human astrocytoma samples by immunohistochemistry and further analyzed the correlation between ATP2A2 expression and clinicopathological characteristics of these astrocytoma patients. We also investigated the effect of ATP2A2 overexpression on the growth of GBM cells in vitro and in a mouse xenograft model.

\section{Materials and methods}

Tissue specimens. In total, 109 formalin-fixed and paraffinembedded surgically resected astrocytic tumor specimens (84 primary ones and 17 secondary GBM and 8 AA developed from DA) were acquired from the Department of Pathology, Changzheng Hospital, Second Military Medical University, Shanghai, China. Samples of oligodendrogiomas with IDH1 mutation $(n=20)$ and pilocytic astrocytomas (PAs) $(n=20)$ without IDH1 mutation were also acquired to further explore the relation between ATP2A2 expression and IDH1 mutation. These specimens were archived between January, 2007 and December, 2014. All analyzed brain tumors were subjected to consensus review by two neuropathologists (Wei-Qing Li, and Hui-Min Liu) according to the WHO Classification (1). Table I lists the types of brain tumors analyzed, including DA $(n=39)$, AA $(n=19)$ and GBM $(n=51)$. There were $61(60.0 \%)$ males and $48(40.0 \%)$ females with a median age of 45 (range, 5-73) years. No patients had received preoperative chemo- or radiotherapy. The average period of follow-up was $27.6 \pm 20.5$ (range, 1-90) months. Tumor size, location, extent of surgical resection, and postoperative consolidated treatment were recorded (Table I). In addition, normal brain tissue samples were obtained from patients undergoing decompressive craniectomy and patients undergoing selective temporal lobe resection for intractable epilepsy.

Acquisition of all tissue specimens was approved by the local ethics committee at the authors' affiliated institution and was carried out in accordance with established institutional and national ethical guidelines regarding use of human tissues for research.

Immunohistochemical staining. Formalin-fixed, paraffinembedded,3- $\mu \mathrm{m}$ tissue sections were cut with 3 adjacent sections chosen from each sample for routine immunohistochemical staining. After deparaffinization in xylol and rehydration in gradient ethanol, sections were immersed in $1 \mu \mathrm{M}$ EDTA buffer $(\mathrm{pH} \mathrm{8.0)}$ and microwaved for $20 \mathrm{~min}$ for antigen retrieval. Endogenous peroxidase was inactivated by $3 \%$ methanolic hydrogen peroxide solution for $30 \mathrm{~min}$. Non-specific binding was blocked by incubation with non-immune serum for $30 \mathrm{~min}$ followed by overnight incubation at $4^{\circ} \mathrm{C}$ with anti-ATP2A2 (Abcam, San Diego, CA, USA), anti-IDH1 and anti-GFAP antibodies (Maxin Biotechnology, Fujian, China). After staining with the DAB kit (Maxin Biotechnology), the slides were counterstained with hematoxylin.

For evaluating ATP2A2 and IDH1 mutation immunoreactivity, two experienced pathologists examined representative visual fields (x200 magnification) independently to identify positively stained tumor cells. The intensity of positive staining was scored using a scale from 0 to 3 ( 0 for no immunostaining, 1 for light-brown color, 2 for medium-brown color, and 3 for dark-brown color). The percentage of positive staining cells was also scored ( 0 , no staining; 1 , positive staining in $<25 \%$ of the tumor cells; 2 , positive staining in $25-75 \%$ of the tumor cells; and 3 , positive staining in $>75 \%$ of the tumor cells). The percentage of cells showing positive staining with the antibodies was calculated in 5 high-powered fields. The two scores were then multiplied, and the results were regarded as the expression score of the sample. All discrepancies in scoring were reviewed, and a consensus was reached. Samples were scored totally as follows: strong $(+++$, total score $=6)$, moderate $(++$, total score $=4-5)$, weak $(+$, total score $=1-3)$, and null $(-$, total score $=0)$. ATP2A2 was recorded as high expression $(++$ and +++$)$, and low or negative expression $(+$ and -) according to the rate of labeled tumor cells and cytoplasm staining intensity.

Cell culture and lentiviral infections. Human glioblastoma cell line U251MG was obtained from the Cell Bank, Type Culture Collection, Chinese Academy of Sciences (CBTCCCAS, Shanghai, China) and cultured at $37^{\circ} \mathrm{C}$ in a humidified atmosphere containing $5 \% \mathrm{CO}_{2}$ in Dulbecco's modified Eagle's medium (DMEM) containing 10\% fetal bovine serum (FBS) supplemented with $100 \mathrm{U} / \mathrm{ml}$ penicillin and $100 \mu \mathrm{g} / \mathrm{ml}$ streptomycin.

The lentivirus vector expressing ATP2A2, pcDNA3.1SERCA2 ATPase, was constructed using the pcDNA3.1 (-)-3Flag-Myc-His expression system according to the manufacturer's instructions (Sunbio Medical Biotechnology, Shanghai, China). The recombinant lentiviruses were produced by co-transfecting human embryonic kidney (HEK) 293T cells using Lipofectamine 2000 according to the manufacturer's protocol (Invitrogen, Carlsbad, CA, USA). U251MG cells were infected with lentiviruses overexpressing ATP2A2 and then selected with $800 \mu \mathrm{g} / \mathrm{ml} \mathrm{G} 418$ (Invitrogen) in complete medium for 20-30 days. Stable U251MG cells were maintained in $\alpha$-MEM containing $10 \%$ FBS and $800 \mu \mathrm{g} / \mathrm{ml} \mathrm{G} 418$. Total cellular RNA and proteins were extracted at 10 days after transfection for further analysis.

Quantitative real-time $R T$-PCR. For quantitative real-time RT-PCR, total cellular RNA was extracted with TRIzol (Invitrogen). Reverse transcription was performed using a Reverse Transcriptase kit (Invitrogen) according to the manufacturer's instructions. The expression of $A T P 2 A 2$ mRNA was 
Table I. Clinicopathological characteristics of 109 diffuse astrocytic tumor patients.

\begin{tabular}{|c|c|}
\hline Variables & $\mathrm{N}(\%)$ \\
\hline \multicolumn{2}{|l|}{ Gender } \\
\hline Male & $61(60.0)$ \\
\hline \multicolumn{2}{|l|}{ Age (years) ${ }^{\mathrm{a}}$} \\
\hline$\geq 55$ & $67(61.5)$ \\
\hline \multicolumn{2}{|l|}{ Tumor site } \\
\hline Temporal lobe & $16(14.7)$ \\
\hline Parietal lobe & $46(42.2)$ \\
\hline Frontal lobe & $31(28.4)$ \\
\hline Occipital lobe & $16(14.7)$ \\
\hline \multicolumn{2}{|l|}{ Tumor size } \\
\hline$>4 \mathrm{~cm}$ & $61(56.0)$ \\
\hline \multicolumn{2}{|c|}{ Extent of resection } \\
\hline Total & $91(83.5)$ \\
\hline Subtotal & $18(16.5)$ \\
\hline \multicolumn{2}{|l|}{ Radiotherapy } \\
\hline Yes & $47(43.1)$ \\
\hline \multicolumn{2}{|l|}{ Chemotherapy } \\
\hline Yes & $60(55.0)$ \\
\hline \multicolumn{2}{|l|}{ WHO grade ${ }^{b}$} \\
\hline II & $39(35.8)$ \\
\hline III & $19(17.4)$ \\
\hline IV & $51(46.8)$ \\
\hline
\end{tabular}

aPatient age refers to age at which the study sample was obtained. ${ }^{\mathrm{b}}$ Tumors were graded according to the World Health Organization (WHO) criteria.

normalized against $\beta$-actin mRNA. The comparative threshold cycle (ct) method was used, and the fold difference $=2^{-(\Delta c t \text { of }}$ target gene - $\Delta$ ct of reference). Quantitative real-time PCR was performed using the SYBR Premix Ex Taq ${ }^{\mathrm{TM}}$ kit (Takara, Kyoto, Japan) on the Takara TP800 System. The PCR was run at $95^{\circ} \mathrm{C}$ for $15 \mathrm{sec}, 40$ cycles of $95^{\circ} \mathrm{C}$ for $5 \mathrm{sec}$ and $60^{\circ} \mathrm{C}$ for $30 \mathrm{sec}$. The sequences of the PCR primers used were as follows: ATP2A2, 5'-CTCGGATCCAACACTACAGGTGTTGAATGG-3' (sense), and 5'-CGGAATTCATGCGCAGTGATAAATTGAC-3' (antisense); $\beta$-actin, 5'-CGTGACATTAAG GAGAAGCTG-3' (sense), and 5'-CTAGAAGCATTTGCGGTGGAC-3 (antsense).

Western blot assays. Cellular lysates were prepared of U251MG cells using RIPA lysis buffer. Proteins were quantified using the Bradford method and samples were resolved on $10 \%$ SDS denatured polyacrylamide gel. Immunoblotting was performed as previously described (18) and the following antibodies were used: mouse anti-flag antibody (Sigma, St. Louis, MO, USA), rabbit anti-SERCA2 ATPase antibody (Abcam), and goat anti-mouse IgG and goat anti-rabbit IgG (all from Santa Cruz Biotechnology, Santa Cruz, CA, USA). The
LabWorks $^{\text {TM }}$ Image Acquisition and Analysis Software (UVP) was used for densitometric analysis.

Cell proliferation and clonogenic assays. For cell proliferation studies, cells were plated onto 96 -well plates at $2 \times 10^{3}$ cells/well and cultured overnight to allow cell attachment. The number of viable cells was determined by the 3-(4,5-dimethylthiazol2-yl)-2,5-diphenyltetrazolium (MTT) bromide colorimetric assays at daily intervals (24, 48, 72, 96 and 120 h). For soft agar clonogenic survival assays, GBM cells were plated onto the top agar at $2.0 \times 10^{2}$ cells per well and grew for 14 days at $37^{\circ} \mathrm{C}$. Colonies were visualized using the cell staining Giemsa solution (Chemicon, Millipore, Billerica, Danvers, MA, USA). An aggregate of $\geq 50$ cells was considered a colony and the number of colonies was counted under the microscope. Each experiment was carried out in triplicate and at least three times independently.

Apoptosis assays. Cells were harvested 8 days after lentiviral infection and washed once with phosphate-buffered saline (PBS), trypsinized, and washed again in PBS with $2 \% \mathrm{FBS}$ and resuspended in binding buffer containing $10 \mathrm{mM}$ HEPES (pH 7.4); $2.5 \mathrm{mM} \mathrm{CaCl}_{2}$, and $140 \mathrm{mM} \mathrm{NaCl}$, and stained with Annexin V-R-PE and 7-AAD according to the manufacturer's protocol (Southern Biotech, Birmingham, AL, USA). Stained cells were analyzed on a FACSCalibur flow cytometer (BD Biosciences) using CellQuest software, and the Mod-Fit program (Verity Software House Inc., Topsham, ME, USA) was used to analyze apoptosis. Each experiment was conducted in triplicate and at least three times independently.

Wound healing assays. The scratch assay was performed to investigate the effect of ATP2A2 overexpression on the migration of GBM cells. Briefly, cells were seeded in 6-well plates at a density of $2 \times 10^{5}$ cells/well. When the cells were 90-100\% confluent, the monolayer was scratched manually with a plastic pipette tip across the diameter of each plate, and after two washes with PBS, the wounded cellular monolayer was allowed to heal for $96 \mathrm{~h}$. Images of central wound edges were taken at time 0 and at the indicated time-points using PowerShot G10 camera (Canon, Tokyo, Japan). Cell migration was observed by microscopy at 24, 48, 72 and $96 \mathrm{~h}$.

Xenograft studies. Forty-five female BALB/c-nu mice, 7-8 weeks of age, were obtained from the Institute of Zoology, Chinese Academy of Sciences, Shanghai, China and kept under specific pathogen-free conditions in accordance with procedures and guidelines set by the Institutional Animal Care and Use Committee (IACUC) of the Second Military Medical University, Shanghai, China. The study protocol was approved by the local institutional review board. Each mouse was inoculated subcutaneously in the forelimb with $1 \times 10^{7} \mathrm{U} 251$ cells in $0.2 \mathrm{ml}$ of medium. After 5 days, mice were randomized to receive twice weekly intratumoral injections $(30 \mathrm{ml} /$ mouse at 3-5 sites) of Lenti-ATP2A2 (pcDNA3.1-SERCA2 ATPase, $5 \times 10^{9} \mathrm{TU} / \mathrm{ml}$ ), or intratumoral injections of control lentiviruses $\left(5 \times 10^{9} \mathrm{TU} / \mathrm{ml}\right)$, or vehicle control. Tumor size was measured every 3 days in two dimensions using a caliper, and tumor volume $\left(\mathrm{mm}^{3}\right)$ was calculated using the formula $\mathrm{V}=0.5 \mathrm{x}$ larger diameter $\mathrm{x}$ (smaller diameter) ${ }^{2}$. Tumor volume was normalized 


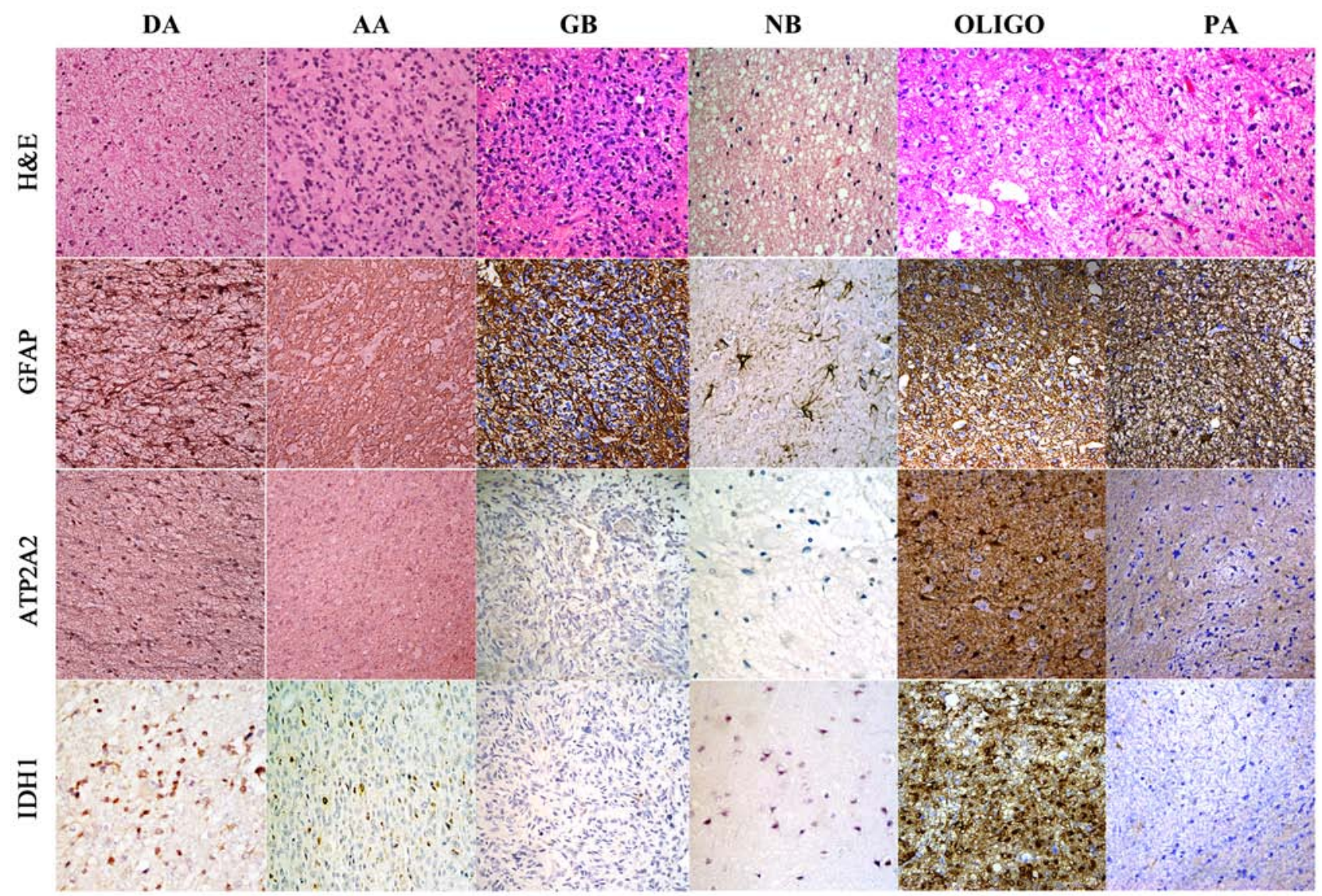

Figure 1. ATP2A2 expression in astrocytoma samples. Immunohistochemistry in normal brain shows that weak ATP2A2 is mainly expressed in some of the neurons, but not in the astrocytes. Stronger staining of ATP2A2 is noted in low-grade astrocytoma (DA) and oligodendrogioma rather than high-grade astrocytomas (AA and glioblastoma) and PA. DA, diffuse astrocytoma; AA, anaplastic astrocytoma; GBM, glioblastoma multiforme; NB, normal brain; OLIGO, oligodendrogioma; PA, pilocytic astrocytoma.

against the initial volume before intratumoral injection for plotting the curve of astrocytoma cell growth rate. Four weeks post injection, 6 mice from each group were randomly selected and sacrificed for weighing and photographing.

Statistical analysis. All statistical analysis and graphs were performed with the SPSS 22.0 analysis software (SPSS Inc., Chicago, IL, USA). The correlation between ATP2A2 expression and clinicopathologic characteristics was analyzed using the Kruskal-Wallis test and Chi-square test. The post hoc Dunn's test of multiple comparisons was used after the Kruskal-Wallis test to examine the sample contrasts between individual sample pairs. Besides, the correlation between ATP2A2 expression and IDH1 mutation in secondary glioblastoma and AA developed from DA was investigated by the McNemar test. The difference of ATP2A2 expression in oligodendrogliomas with IDH1 mutation and PAs without IDH1 mutation was investigated by Chi-square test. Survival was defined from the date of surgical diagnosis to death from any cause. Correlation of ATP2A2 expression with survival was determined using the Kaplan-Meier product-limit method, and differences between survival curves were tested using the log-rank test. For other experiments, differences between groups were measured by Student's t-test, and for comparing means of more than 2 groups, one-way ANOVA was used. P-values $<0.05$ were considered as statistically significant.
Table II. ATP2A2 immunoexpression based on staining intensity scores in diffuse astrocytic tumor according to tumor grade $(n=109)$.

\begin{tabular}{lrrrrrr}
\hline \multicolumn{6}{c}{ Staining intensity scores of ATP2A2 } \\
\cline { 2 - 7 } & $\begin{array}{c}+ \\
(\mathrm{n}=32)\end{array}$ & $\begin{array}{c}++ \\
(\mathrm{n}=30)\end{array}$ & $\begin{array}{c}++++ \\
(\mathrm{n}=32)\end{array}$ & Total & P-value \\
& & & & & & 0.036 \\
WHO grade & & 6 & 10 & 14 & 39 & \\
II & 1 & 8 & 7 & 3 & 19 & \\
III & 19 & 14 & 9 & 9 & 51 & \\
IV & &
\end{tabular}

Tumors were graded by the World Health Organization (WHO) criteria.

\section{Results}

ATP2A2 is variably expressed in astrocytoma tissues and correlated with astrocytoma grade. We found weak staining of ATP2A2 in some of the neurons but not in the astrocytes of normal brains (Fig. 1). ATP2A2 was not expressed in $26.6 \%$ $(29 / 109)$ of the patients, mildly positive in $25.7 \%$ (28/109), moderately positive in $23.9 \%$ (26/109) and strongly positive in $23.9 \%(26 / 109)$ of the patients (Table II). It was further 
Table III. ATP2A2 immunoexpression in diffuse astrocytic tumor according to tumor grade $(n=109)$.

\begin{tabular}{lcccc}
\hline & $\begin{array}{c}\text { Low ATP2A2 } \\
\text { expression }\end{array}$ & $\begin{array}{c}\text { High ATP2A2 } \\
\text { expression }\end{array}$ & Total & P-value \\
\hline WHO grade & & & & 0.042 \\
II & 15 & 24 & 39 & \\
III & 9 & 10 & 19 & \\
IV & 33 & 18 & 51 & \\
\hline
\end{tabular}

examined whether ATP2A2 expression was associated with astrocytoma grades. ATP2A2 was positive in $76.9 \%$ (30/39) of grade II patients, $94.7 \%(18 / 19)$ of grade III patients and $62.8 \%$ $(32 / 51)$ of grade IV patients $(\mathrm{P}=0.036$, Table II). Moreover, $35.3 \%(18 / 51)$ of grade IV astrocytoma patients showed high positive staining of ATP2A2, which was significantly lower than that of grade II $(61.5 \%, 24 / 39)$ and grade III $(52.6 \%, 10 / 19)$ astrocytoma patients $(\mathrm{P}=0.042$, Table III). The staining pattern of ATP2A2 in different grades of astrocytomas is illustrated in Fig. 1. As shown in Table IV, statistical analysis revealed a close correlation between ATP2A2 expression and WHO grade of astrocytomas $(\mathrm{P}=0.025)$. Furthermore, the results of the post hoc Dunn's test used after the Kruskal-Wallis test showed that the difference of ATP2A2 expression rate in grade IV and grade II was significant $(\mathrm{P}=0.015)$. However, there was no significant difference in the ATP2A2 expression between grade II glioma and grade III glioma $(\mathrm{P}=0.765)$, and grade III glioma and grade IV glioma $(\mathrm{P}=0.105)$. The above findings indicated that, despite variable expression of ATP2A2 in astrocytoma tissues, the most aggressive form of astrocytomas, GBM, exhibits noticeably lower expression of ATP2A2 compared to astrocytoma of lower grades. In addition, high ATP2A2 expression level is also seen in the patients with the secondary glioblastoma and AAs developed from DAs $(56.0 \%, 14 / 25)$, which is similar to the relationship between IDH1 mutation and the secondary glioblastoma and AAs developed from DAs $(44.0 \%, 11 / 25)(\mathrm{P}=0.508)$. To further illustrate the correlation of high ATP2A 2 expression and IDH1 mutation, ATP2A2 expression was also examined in oligodendrogiomas with IDH1 mutation and PAs without IDH1 mutation. The result supported that high ATP2A2 was more frequently observed in astrocytomas with IDH1 mutation $(15 / 20)$ than in those without IDH1 mutation $(3 / 20)$ (Chi-square test, $\mathrm{P}<0.001$ ) (Fig. 1).

ATP2A2 expression correlates with survival of astrocytoma patients. The Kaplan-Meier distribution of survival is shown

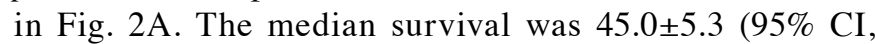
34.7-55.3) months in patients with high ATP2A2 expression and $16.0 \pm 5.0(95 \% \mathrm{CI}, 6.3-25.7)$ months in patients with low ATP2A 2 expression $(\mathrm{P}<0.0001)$. There was a significant correlation between ATP2A2 expression and survival. We further analyzed the survival of high grade astrocytoma patients stratified by ATP2A2 expression. High grade astrocytoma patients with high ATP2A2 expression showed markedly longer survival (median, 31.0 \pm 4.9 months; 95\% CI, 21.4-40.7) than
Table IV. ATP2A2 immunoexpression in low and high grade diffuse astrocytic tumor $(\mathrm{n}=109)$.

Low ATP2A2 High ATP2A2 Total P-value expression expression

\begin{tabular}{lllll}
\hline Glioma grade & & & & 0.025 \\
Low & 15 & 24 & 39 & \\
High & 42 & 28 & 70 & \\
\hline
\end{tabular}

those with low ATP2A2 expression (median, 13.0 11.6 months; 95\% CI, 9.9-16.1) ( $\mathrm{P}=0.027$, Fig. 2B). Low grade astrocytoma patients with lower ATP2A2 expression showed significantly shorter survival (47.0 \pm 7.2 months, 95\% CI, 32.8-61.2) than those with higher ATP2A2 expression (67.0 115.8 months, 95\% CI, 36.0-98.0) ( $\mathrm{P}=0.039$, Fig. 2C). Besides, we examined whether the survival time was influenced by postoperative adjuvant therapy (radiotherapy and chemotherapy) in astrocytoma patients of different grades with different ATP2A2 expression levels (Fig.3). As shown in Fig. 3H, only in high grade astrocytoma patients with high ATP2A2 levels, those who received chemotherapy showed significantly longer survival (34 \pm 5.7 months, 95\% CI, 22.7-45.3) than those who did not $(19 \pm 6.7$ months, $95 \%$ CI, 5.8-32.2) $(\mathrm{P}=0.021)$.

ATP2A2 overexpression suppresses the clonogenic growth and migration of GBM cells. The above findings suggest that ATP2A2 may play a hitherto undiscovered role in the oncogenesis and development of astrocytomas. To decipher the role of ATP2A2 in astrocytoma genesis, we infected GBM U251MG cells with lentiviral vectors overexpressing ATP2A2 (Lenti-ATP2A2), which resulted in a $132 \%$ increase in the mRNA transcript levels and $163 \%$ increase in the protein levels of ATP2A2 (Fig. 4A and B). The MTT assays showed that ATP2A2 overexpression significantly suppressed the growth of U251MG cells 72 and $96 \mathrm{~h}$ post transfection $(\mathrm{P}<0.05$ or $<0.001)$, with a $15.2 \pm 0.7 \%$ reduction in the growth of $\mathrm{U} 251 \mathrm{MG}$ cells at $96 \mathrm{~h}$ (Fig. 4C). Additionally, the clonogenic assays showed that ATP2A2 overexpression caused a 54.2 $\pm 1.9 \%$ decrease in the number of colonies (Fig. 4D) $[\mathrm{P}=0.0004$ vs. Lenti-green fluorescent protein (Lenti-GFP)]. We further investigated whether the growth-inhibitory effect of ATP2A2 was associated with induction of apoptosis of GBM cells. Our flow cytometric analysis revealed that ATP2A2 overexpression was associated with a significant increase in the percentage of apoptotic U251MG cells (Lenti-ATP2A2, 48.4 $\pm 3.3 \%$ vs. Lenti-GFP, $1.7 \pm 0.2 \%, \mathrm{P}=0.0008$ ) (Fig. 4E).

Moreover, we sought to investigate whether ATP2A2 impacted on the migration of GBM cells. Our wound healing assays revealed that ATP2A2 overexpression markedly suppressed the migration of U251MG cells (Fig. 5).

ATP2A2 overexpression inhibits GBM xenograft growth in mice. It was examined whether ATP2A2 overexpression suppressed the growth of GBM xenografts in mice. We found that, compared with control xenografts expressing GFP, tumors stably overexpressing ATP2A2 were markedly 
A

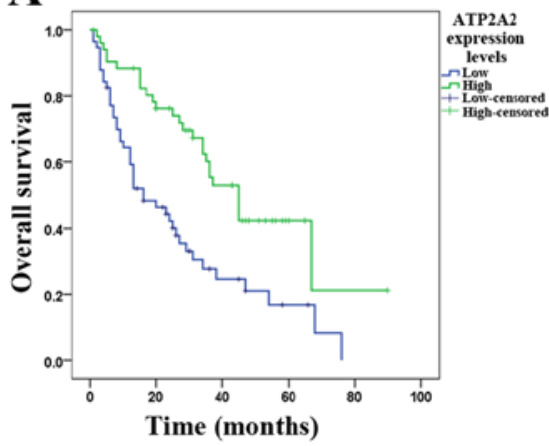

B

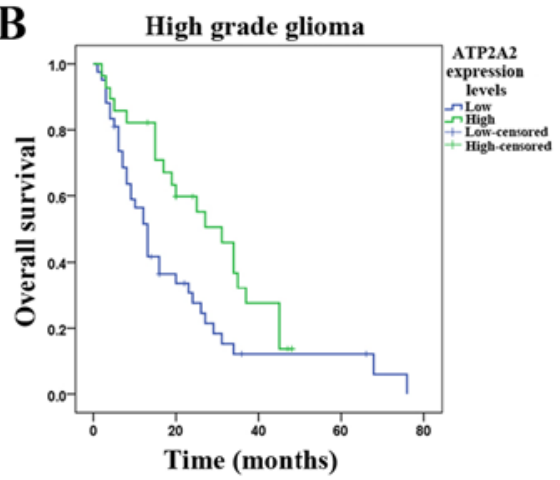

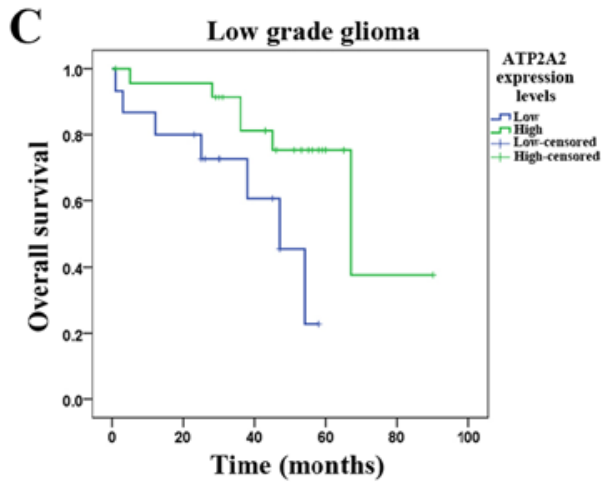

Figure 2. Kaplan-Meier postoperative survival curve for astrocytoma patients stratified by ATP2A2 expression. Low and high ATP2A2 expression is defined in Materials and methods. (A) The median overall survival of astrocytoma patients with high and low ATP2A2 expression is $45 \pm 5.3$ (95\% CI, 34.7-55.3) and $16 \pm 5.0$ (95\% CI, 6.3-25.7), respectively (log-rank test, P<0.0001). (B) High grade astrocytoma patients with high ATP2A2 expression show markedly longer survival ( $31 \pm 4.9$ months, CI, 21.4-40.7) than those with low ATP2A2 expression (13 \pm 1.6 months, CI, 9.9-16.1) (P=0.027). (C) Low grade astrocytoma patients with lower ATP2A2 expression show significantly shorter survival (47.0 \pm 7.2 months, 95\% CI, 32.8-61.2) than those with higher ATP2A2 expression (67.0 \pm 15.8 months, $95 \%$ CI, 36.0-98.0) $(\mathrm{P}=0.039)$.

A

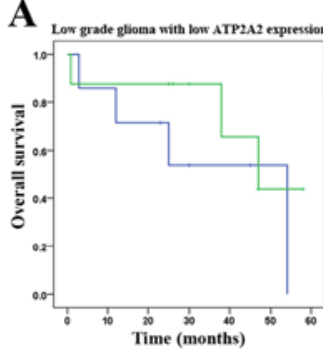

E

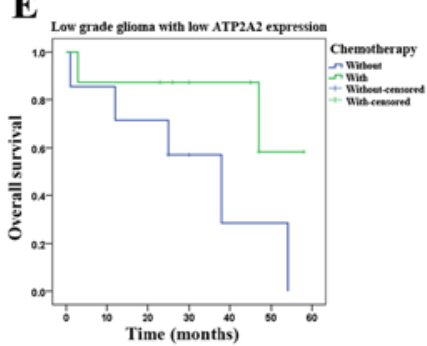

B

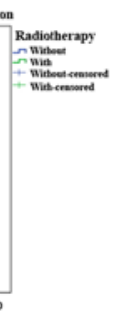

F
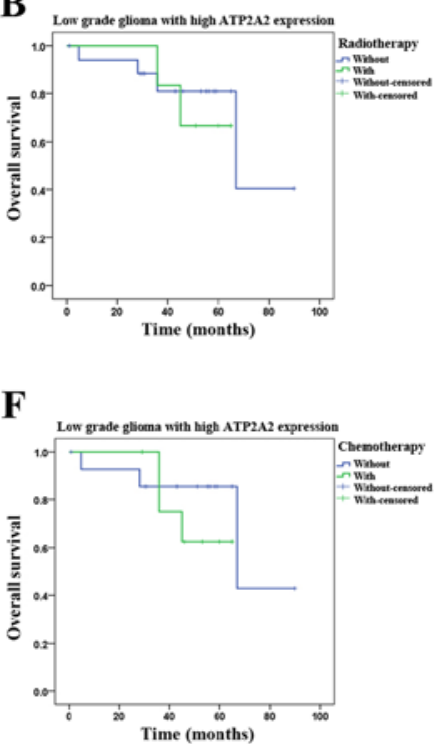

C

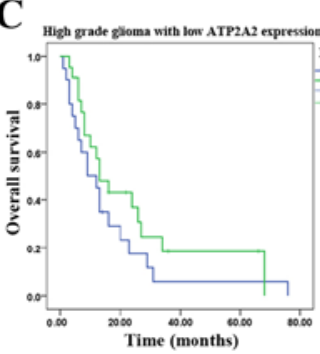

G

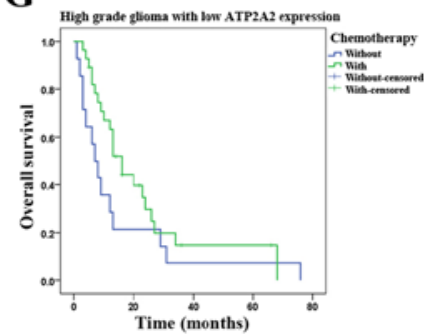

D
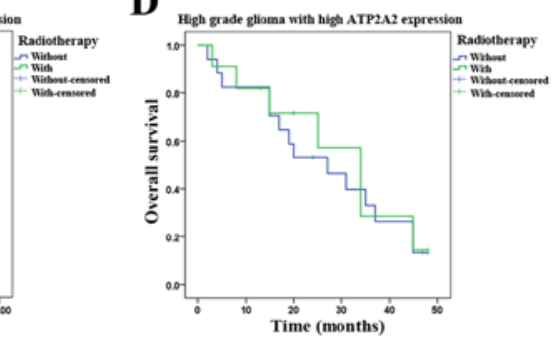

H

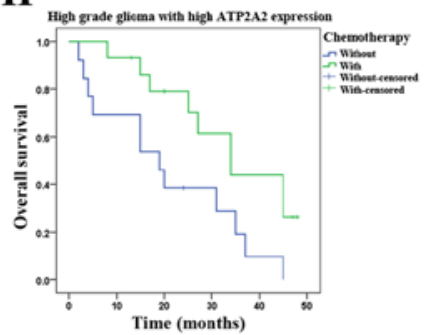

Figure 3. Association of postoperative adjuvant therapy with overall survival in astrocytoma patients of different grades stratified by ATP2A2 expression. Only in high grade astrocytoma patients with high ATP2A2 levels, those who received chemotherapy showed significantly longer survival (34 \pm 5.7 months, $95 \%$ CI, 22.7-45.3) than those who did not receive chemotherapy (19 \pm 6.7 months, 95\% CI, 5.8-32.2) $(\mathrm{P}=0.021)$.

smaller in size 3 weeks after xenograft implantation (Fig. 6). The mean tumor volume was $81.6 \pm 21.5 \mathrm{~mm}^{3}$ in xenografts overexpressing ATP2A 2 and $238.2 \pm 65.2 \mathrm{~cm}^{3}$ in the control xenografts respectively at week 4 post inoculation $(\mathrm{P}<0.05)$.

\section{Discussion}

Comprehensive elucidation of genetic alterations in astrocytomas may provide novel therapeutic targets, and diagnostic as well as prognostic markers for the disease. Furthermore, detailed delineation of prognostic markers for astrocytomas, especially GBMs, may lead to the identification of subsets of astrocytoma patients who may have better prognosis in response to adjuvant or neoadjuvant chemotherapies or targeted therapies. Here, we characterized the expression of ATP2A2 in astrocytoma tissues and documented for the first time that ATP2A2 showed altered expression in astrocytoma tissues. We found that ATP2A2 was expressed in the majority $(73.4 \%$, $80 / 109)$ of the patients and more than half $(62.8 \%, 32 / 51)$ of grade IV astrocytoma patients expressed ATP2A2. Two thirds $(61.5 \%, 24 / 39)$ of grade II astrocytoma patients exhibited high ATP2A2 expression while only approximately one third $(35.3 \%, 18 / 51)$ of grade IV astrocytoma patients showed high ATP2A2 expression. Our statistical analysis further revealed a close correlation between ATP2A2 expression and WHO grade of astrocytomas. These findings implicate ATP2A2 participates in the oncogenesis in astrocytomas.

ATP2A2 acts as a critical component of several signaling pathways that have been demonstrated to be aberrant in astrocytoma. Genetic alterations occur frequently in the RTK/ 
A

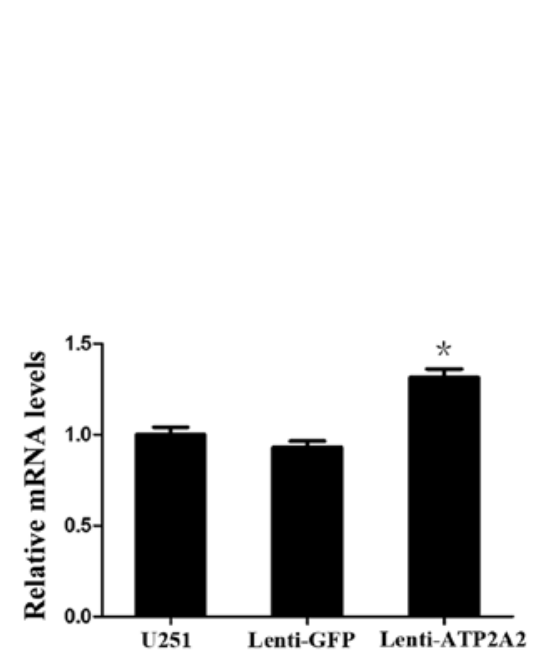

C

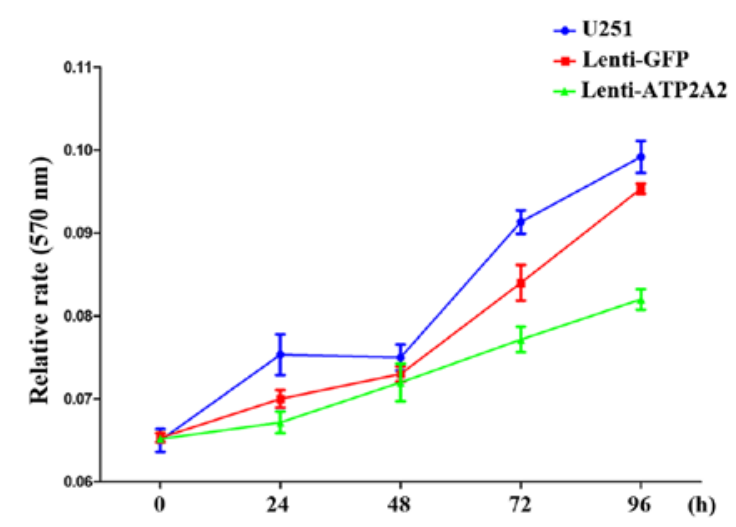

B
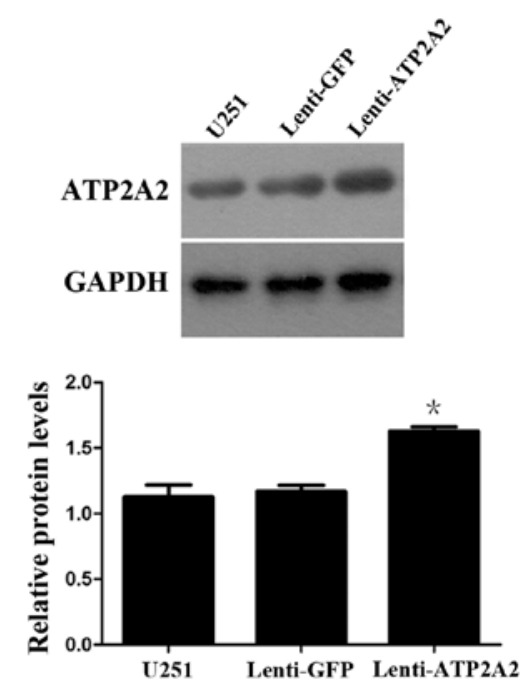

D
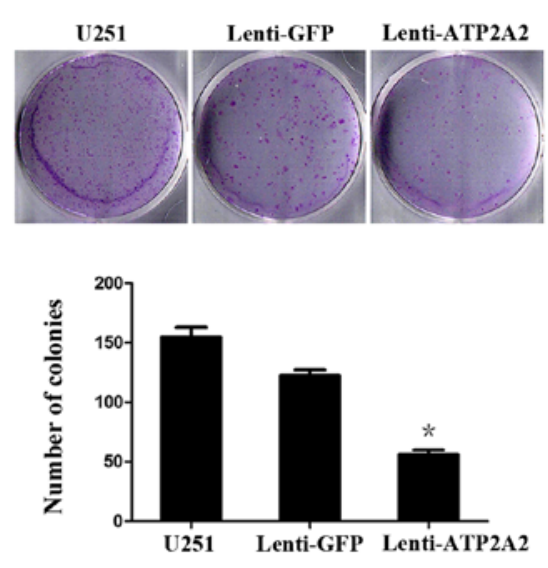

E

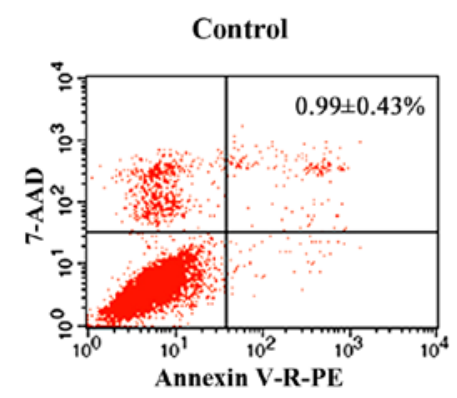

Lenti-GFP

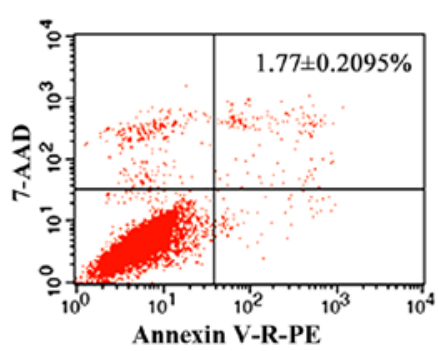

Lenti-ATP2A2

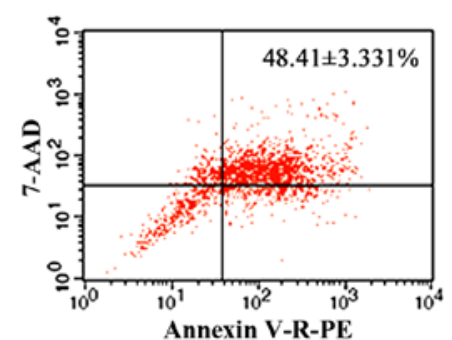

Figure 4. ATP2A2 overexpression was determined by western blot and real-time RT-PCR assays. U251MG cells were transfected with lentiviral vectors overexpressing ATP2A2 (Lenti-ATP2A2) or control vector (Lenti-GFP). The ATP2A2 mRNA transcript levels (A) and ATP2A2 protein levels (B) were significantly increased in U251MG cells transfected with Lenti-ATP2A2. Cellular proliferation was examined by the MTT assays (C) and soft agar assays (D). Apoptosis of U251 cells was examined by flow cytometry (E). Data in (A-D) are shown as mean \pm SD of at least three independent experiments. Data in (E) are representative of at least three independent experiments. ${ }^{~} \mathrm{P}<0.05$ vs. Lenti-GFP or non-transfected controls.

RAS/PI-3K signaling pathway, the p53 signaling pathway and the RB signaling pathway (17). RAS signaling and calcium signaling converge at Raf1 (18) while calcium plays a pivotal role in p53 signaling (19) and $\mathrm{Ca}^{2+}$ and $\mathrm{CaM}$-dependent signaling is required for RB phosphorylation $(20,21)$. ATP2A2 expression is downregulated in cancer of the lung and head and neck squamous cell cancer, but upregulated in the colorectal cancer, liposarcoma and prostate cancer $(15,22-25)$.
Our demonstration of aberrant expression of ATP2A2 in astrocytoma tissues further adds ATP2A2 in a growing list of malignant cancers in humans. Besides, high ATP2A2 expression was associated with better overall survival of astrocytoma patients. Our study also evaluated whether ATP2A2 could be used as a prognostic marker for identifying a subset of astrocytoma patients responding preferentially to particular therapies. The result indicates that high grade astrocytoma patients 


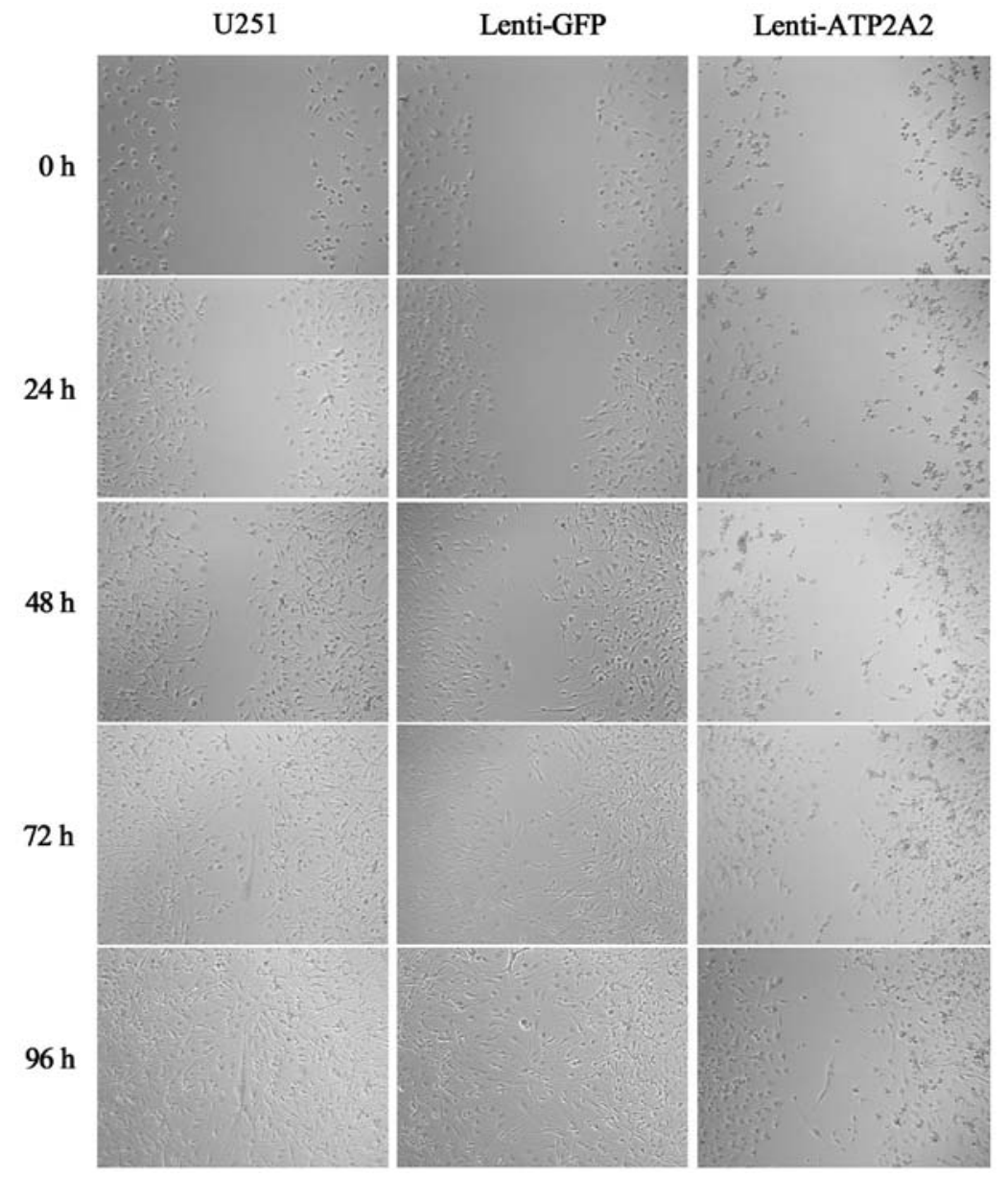

Figure 5. Effects of ATP2A2 overexpression on GBM cell invasion and migration. U251MG cells were transfected as described in Fig. 4 and wound scratch assays were performed as detailed in Materials and methods. Images of migrated cells were taken at $0,24,48,72$ and $96 \mathrm{~h}$ and these images are representative of at least 3 independent experiments.

A

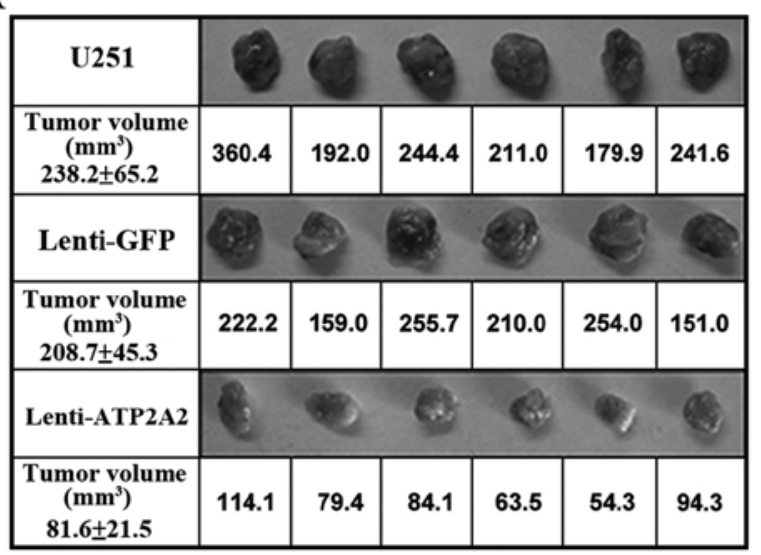

B

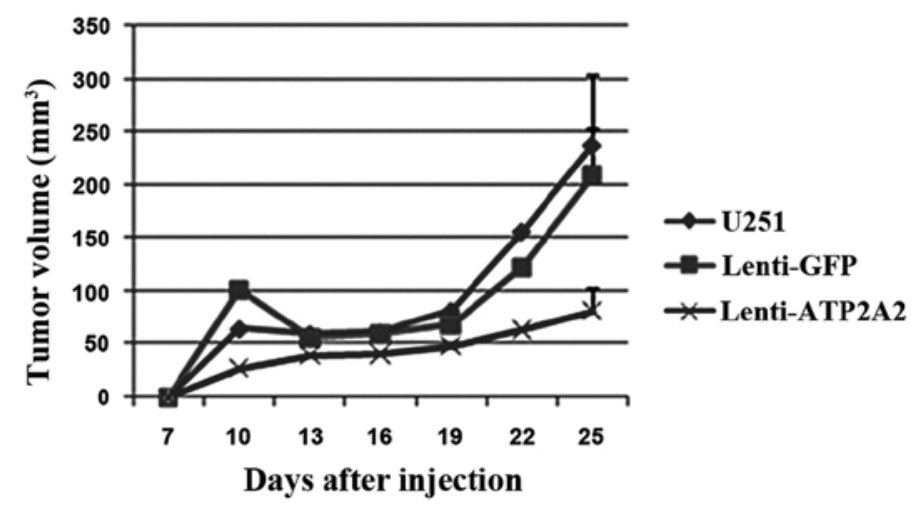

Figure 6. ATP2A2 overexpression suppresses xenograft tumor growth. (A) The effect of ATP2A2 overexpression on xenograft tumor growth was examined in SCID mice. U251MG cells stably overexpressing ATP2A2 were injected into the mice. Four weeks after injection, the tumors were dissected and captured. (B) Tumor size was measured every three days and data are shown as mean $\pm \mathrm{SD}$. ${ }^{*} \mathrm{P}<0.05$ vs. Lenti-GFP or non-transfected controls.

with high ATP2A2 expression level are more sensitive to the postoperative chemotherapy. Another substantial result of our study is that high ATP2A2 expression shared a similar distribution pattern with IDH1 mutation in secondary GBM, AA developed from DA, oligodendroglioma and PA, suggesting that ATP2A2 may possess a similar prognostic value as IDH1 in astrocytoma (26). We hypothesized that the alteration of energy metabolism due to IDH1 mutation (27) may affect the expression and function of ATP2A2. The aforementioned outcomes are consistent with findings from previous studies 
on the role of ATP2A2 in other tumor types $(22,23)$, implying that ATP2A2 expression may be a potential molecular marker for predicting the prognosis of astrocytoma patients.

In this study, we provide further evidence that increased ATP2A2 expression inhibited the clonogenic growth of GBM cells in vitro, probably via induction of apoptosis. Additionally, we observed that ATP2A2 overexpression depressed the migration of GBM cells in an in vitro wound healing assay. Moreover, tumor xenograft growth was noticeably suppressed by intratumoral injection of lentiviral vectors overexpressing ATP2A2. This may partially explain the survival benefit of high ATP2A2 expression in astrocytoma patients. However, it still needs to be further validated by additional studies.

Sarco (endo)plasmic reticulum (SER) $\mathrm{Ca}^{2+}$ ATPases are a highly conserved family of $\mathrm{Ca}^{2+}$ pumps. SERCA2 is implicated in certain cancers. Another isoform, SERCA3, encoded by the ATP $2 A 3$ gene, has been shown to be downregulated in colon cancer, lung adenocarcinoma and head and neck squamous cell cancer $(11,28,29)$. Both proteins are involved in maintaining $\mathrm{Ca}^{2+}$ homeostasis, which is critical to the normal functioning of a plethora of cellular processes ranging from cellular proliferation to apoptosis by modulating cellular signaling pathways (30), and the aberration of some of these $\mathrm{Ca}^{2+}$-mediated signaling pathways is implicated in tumorigenesis and tumor progression, such as metastasis, invasion and angiogenesis (31). ER $\mathrm{Ca}^{2+}$ levels remain undefined in astrocytoma cells. Astrocytoma cells are known to suffer from low grade of ER stress (32). Agents affecting ER $\mathrm{Ca}^{2+}$ homeostasis such as flavonoids could activate ER stress and induce cell death in glioma cells (33). Kovacs et al (34) found that compared with normal astrocytes, GBM cells exhibit higher resting cytosolic $\mathrm{Ca}^{2+}$ levels. It remains to be elucidated how ATP2A2 induces apoptosis of GBM cells in terms of ER stress. We speculate that in astrocytoma, alteration in ATP2A2 expression may contribute to tumorigenesis by interfering with the balance between cytosolic and ER $\mathrm{Ca}^{2+}$ level, thereby affecting certain $\mathrm{Ca}^{2+}$ signaling pathways, especially the RTK/RAS/PI-3K signaling, p53 signaling and RB signaling pathways (17). However, the exact mechanisms whereby ATP2A2 is involved in oncogenesis and development of astrocytomas require further investigation. In some other studies, the intervention of ATP2A2 expression, either upregulating or inhibiting, could exert influence on the progression of various malignancies $(14,35)$, indicating that ATP2A2 may also function as a therapeutic target for astrocytoma treatment.

In conclusion, we present here direct clinical evidence that ATP2A2 is variably expressed in astrocytoma tissues and its expression correlates with tumor grade, and secondary glioblastoma and AA developed from DA has a propensity to overexpress ATP2A2. Importantly, patients with high ATP2A2 expression show better overall survival, thus identifying a subset of astrocytoma patients with differing prognosis. Furthermore, ATP2A2 overexpression suppresses growth of astrocytoma cells. Although the precise correlation of the altered ATP2A2 expression with astrocytoma grade, molecular subtypes or clinical parameters and the underlying mechanism requires further investigation, our findings implicate ATP2A2 in gliomagenesis and suggest that ATP2A 2 may serve as a prognostic marker identifying astrocytoma patients with differing prognoses.

\section{Acknowledgements}

This study was supported by grants from the Shanghai Municipal Science and Technology Commission (no. 10ZR1439000 to Yi-Ming Li), the National Natural Sciences Fund Project of China (NSFC no. 81101656/H1609 to Yi-Ming Li, NSFC no. 81201987/H1618 to Wei-Qing Li and NSFC no. 30930094 to Yi-Cheng $\mathrm{Lu}$ ).

\section{References}

1. Louis DN, Perry A, Reifenberger G, von Deimling A, FigarellaBranger D, Cavenee WK, Ohgaki H, Wiestler OD, Kleihues P and Ellison DW: The 2016 World Health Organization Classification of Tumors of the Central Nervous System: A summary. Acta Neuropathol 131: 803-820, 2016.

2. Parisot S, Darlix A, Baumann C, Zouaoui S, Yordanova Y, Blonski M, Rigau V, Chemouny S, Taillandier L, Bauchet L, et al: A probabilistic atlas of diffuse WHO grade II glioma locations in the brain. PLoS One 11: e0144200, 2016.

3. Dubrow R, Darefsky AS, Jacobs DI, Park LS, Rose MG, Laurans MS and King JT Jr: Time trends in glioblastoma multiforme survival: The role of temozolomide. Neuro-oncol 15: 1750-1761, 2013.

4. Mesti T and Ocvirk J: Malignant gliomas: Old and new systemic treatment approaches. Radiol Oncol 50: 129-138, 2016.

5. Watanabe T, Nobusawa S, Kleihues $\mathrm{P}$ and Ohgaki H: IDH1 mutations are early events in the development of astrocytomas and oligodendrogliomas. Am J Pathol 174: 1149-1153, 2009.

6. Chow LM, Endersby R, Zhu X, Rankin S, Qu C, Zhang J Broniscer A, Ellison DW and Baker SJ: Cooperativity within and among Pten, p53, and Rb pathways induces high-grade astrocytoma in adult brain. Cancer Cell 19: 305-316, 2011.

7. Hofer S, Rushing E, Preusser M and Marosi C: Molecular biology of high-grade gliomas: What should the clinician know? Chin J Cancer 33: 4-7, 2014.

8. Bchetnia M, Benmously R, Ben Brick AS, Charfeddine C, Ben Ameur Y, Fajraoui M, Debbiche A, Ben Ayed M, Mokni M, Fenniche S, et al: New mutations of Darier disease in Tunisian patients. Arch Dermatol Res 301: 615-619, 2009.

9. Shi BJ, Feng J, Ma CC, Yan XN, Li WB, Wei YP, Hu G and Wang XL: Novel mutations of the ATP2A2 gene in two families with Darier's disease. Arch Dermatol Res 301: 27-30, 2009.

10. Papp B, Brouland JP, Gélébart P, Kovàcs T and Chomienne C: Endoplasmic reticulum calcium transport ATPase expression during differentiation of colon cancer and leukaemia cells. Biochem Biophys Res Commun 322: 1223-1236, 2004.

11. Wu Y, Palad AJ, Wasilenko WJ, Blackmore PF, Pincus WA, Schechter GL, Spoonster JR, Kohn EC and Somers KD: Inhibition of head and neck squamous cell carcinoma growth and invasion by the calcium influx inhibitor carboxyamido-triazole. Clin Cancer Res 3: 1915-1921, 1997.

12. Baron S, Vangheluwe P, Sepúlveda MR, Wuytack F, Raeymaekers L and Vanoevelen J: The secretory pathway Ca (2+)-ATPase 1 is associated with cholesterol-rich microdomains of human colon adenocarcinoma cells. Biochim Biophys Acta 1798: 1512-1521, 2010.

13. Matsui K, Makino T, Nakano H, Furuichi M, Sawamura D and Shimizu T: Squamous cell carcinoma arising from Darier's disease. Clin Exp Dermatol 34: e1015-e1016, 2009.

14. Bleeker NP, Cornea RL, Thomas DD and Xing C: A novel SERCA inhibitor demonstrates synergy with classic SERCA inhibitors and targets multidrug-resistant AML. Mol Pharm 10: 4358-4366, 2013.

15. Wang L, Li W, Yang Y, Hu Y, Gu Y, Shu Y, Sun Y, Wu X, Shen $Y$ and $\mathrm{Xu} \mathrm{Q}$ : High expression of sarcoplasmic/endoplasmic reticulum $\mathrm{Ca}(2+)$-ATPase $2 \mathrm{~b}$ blocks cell differentiation in human liposarcoma cells. Life Sci 99: 37-43, 2014.

16. Prasad V, Boivin GP, Miller ML, Liu LH, Erwin CR, Warner BW and Shull GE: Haploinsufficiency of Atp2a2, encoding the sarco (endo)plasmic reticulum $\mathrm{Ca}^{2+}$-ATPase isoform $2 \mathrm{Ca}^{2+}$ pump, predisposes mice to squamous cell tumors via a novel mode of cancer susceptibility. Cancer Res 65: 8655-8661, 2005.

17. Cancer Genome Atlas Research Network: Comprehensive genomic characterization defines human glioblastoma genes and core pathways. Nature 455: 1061-1068, 2008. 
18. Yoshiki S, Matsunaga-Udagawa R, Aoki K, Kamioka Y, Kiyokawa E and Matsuda M: Ras and calcium signaling pathways converge at Raf1 via the Shoc2 scaffold protein. Mol Biol Cell 21: 1088-1096, 2010.

19. Sorrentino G, Comel A and Del Sal G: p53 orchestrates calcium signaling in vivo. Cell Cycle 14: 1343-1344, 2015.

20. Takuwa N, Zhou W, Kumada M and Takuwa Y: Ca (2+)-dependent stimulation of retinoblastoma gene product phosphorylation and p34cdc2 kinase activation in serum-stimulated human fibroblasts. J Biol Chem 268: 138-145, 1993.

21. Rasmussen CD and Means AR: Calmodulin is required for cell-cycle progression during G1 and mitosis. EMBO J 8: 73-82, 1989.

22. Korosec B, Glavac D, Rott T and Ravnik-Glavac M: Alterations in the ATP2A2 gene in correlation with colon and lung cancer. Cancer Genet Cytogenet 171: 105-111, 2006.

23. Korosec B, Glavac D, Volavsek M and Ravnik-Glavac M: Alterations in genes encoding sarcoplasmic-endoplasmic reticulum $\mathrm{Ca}(2+)$ pumps in association with head and neck squamous cell carcinoma. Cancer Genet Cytogenet 181: 112-118, 2008.

24. Huang MY, Wang HM, Tok TS, Chang HJ, Chang MS, Cheng TL, Wang JY and Lin SR: EVI2B, ATP2A2, S100B, TM4SF3, and OLFM4 as potential prognostic markers for postoperative Taiwanese colorectal cancer patients. DNA Cell Biol 31: 625-635, 2012.

25. Legrand G, Humez S, Slomianny C, Dewailly E, Vanden Abeele F, Mariot P, Wuytack F and Prevarskaya N: $\mathrm{Ca}^{2+}$ pools and cell growth. Evidence for sarcoendoplasmic $\mathrm{Ca}^{2+}$-ATPases $2 \mathrm{~B}$ involvement in human prostate cancer cell growth control. J Biol Chem 276: 47608-47614, 2001.

26. Karsy M, Neil JA, Guan J, Mahan MA, Colman H and Jensen RL: A practical review of prognostic correlations of molecular biomarkers in glioblastoma. Neurosurg Focus 38: E4, 2015.
27. Reitman ZJ, Duncan CG, Poteet E, Winters A, Yan LJ, Gooden DM, Spasojevic I, Boros LG, Yang SH and Yan H: Cancer-associated isocitrate dehydrogenase 1 (IDH1) R132H mutation and d-2-hydroxyglutarate stimulate glutamine metabolism under hypoxia. J Biol Chem 289: 23318-23328, 2014.

28. Flores-Peredo L, Rodríguez $\mathrm{G}$ and Zarain-Herzberg A: Induction of cell differentiation activates transcription of the Sarco/ Endoplasmic Reticulum calcium-ATPase 3 gene (ATP2A3) in gastric and colon cancer cells. Mol Carcinog 56: 735-750, 2017.

29. Arbabian A, Brouland JP, Apáti Á, Pászty K, Hegedús L, Enyedi Á, Chomienne C and Papp B: Modulation of endoplasmic reticulum calcium pump expression during lung cancer cell differentiation. FEBS J 280: 5408-5418, 2013.

30. Berridge MJ, Bootman MD and Roderick HL: Calcium signalling: Dynamics, homeostasis and remodelling. Nat Rev Mol Cell Biol 4: 517-529, 2003.

31. Monteith GR, McAndrew D, Faddy HM and Roberts-Thomson SJ: Calcium and cancer: Targeting $\mathrm{Ca}^{2+}$ transport. Nat Rev Cancer 7: 519-530, 2007.

32. Johnson GG, White MC and Grimaldi M: Stressed to death: Targeting endoplasmic reticulum stress response induced apoptosis in gliomas. Curr Pharm Des 17: 284-292, 2011.

33. Das A, Banik NL and Ray SK: Flavonoids activated caspases for apoptosis in human glioblastoma T98G and U87MG cells but not in human normal astrocytes. Cancer 116: 164-176, 2010.

34. Kovacs GG, Zsembery A, Anderson SJ, Komlosi P, Gillespie GY, Bell PD, Benos DJ and Fuller CM: Changes in intracellular $\mathrm{Ca}^{2+}$ and $\mathrm{pH}$ in response to thapsigargin in human glioblastoma cells and normal astrocytes. Am J Physiol Cell Physiol 289: C361C371, 2005.

35. Seo JA, Kim B, Dhanasekaran DN, Tsang BK and Song YS: Curcumin induces apoptosis by inhibiting sarco/endoplasmic reticulum $\mathrm{Ca}^{2+}$ ATPase activity in ovarian cancer cells. Cancer Lett 371: 30-37, 2016. 